\title{
Metadiscurso e escrita acadêmica: o papel dos recursos interacionais na construção do discurso das disciplinas de Ciências Políticas e Linguística
}

DOl: http://dx.doi.org/10.21165/el.v50i3.2965

\section{Beatriz Gil'}

\section{Resumo}

O objetivo deste artigo é analisar (i) como o metadiscurso aparece na constituição da escrita acadêmica em disciplinas de Humanidades, em língua inglesa e (ii) se é possível notar algum padrão de semelhança e/ou diferença nas recorrências do metadiscurso entre as disciplinas. Para tanto, foi analisado um corpus de 48 abstracts de artigos de pesquisa publicados em língua inglesa em revistas internacionais Qualis A1 e retirados de quatro periódicos das áreas de Ciência Política e Linguística. Ambos os periódicos de Ciência Política apresentam maior recorrência de hedges e boosters quando comparados com os periódicos de Linguística. Esse dado pode ser explicado à luz das fronteiras disciplinares, uma vez que os primeiros estão em contato mais direto com características e crenças do fazer pesquisa das Ciências Humanas, enquanto os dois últimos com aquelas presentes em Ciências Biológicas e em áreas da Saúde.

Palavras-chave: humanidades; escrita acadêmica; metadiscurso.

1 Universidade Estadual Paulista "Júlio de Mesquita Filho" (UNESP), São José do Rio Preto, São Paulo, Brasil; beatriz_gil24@hotmail.com; https://orcid.org/0000-0002-4534-5366 


\title{
Metadiscourse and academic writing: the role of interactive resources in the construction of Political Science and Linguistic disciplines discourse
}

\begin{abstract}
The purpose of this article is to analyze (i) how the metadiscourse appears in Humanities academic writing in English and (ii) whether it is possible to notice similarities and/ or differences in the recurrences of the metadiscourse among the disciplines. As a methodology, it was analyzed 48 research article abstracts published in four Qualis A1 journals from the areas of Political Science and Linguistics. The Political Science journals show a greater recurrence of hedges and boosters when compared to Linguistics journals. This data can be explained considering the disciplinary boundaries: Political Science journals are in more direct contact with characteristics and beliefs of Human Sciences research, while the Linguistics one's present interface with Biological and Health Sciences.
\end{abstract}

Keywords: humanities; academic writing; metadiscourse.

\section{Introdução}

No contexto acadêmico atual, cujo principal meio de divulgação e compartilhamento de estudos e descobertas é a escrita, sobram iniciativas, cursos e manuais destinados a discutir e "ensinar" as características dessa escrita e como ela deve ser feita. Um dos pressupostos mais recorrentes é o da impessoalidade e da neutralidade na academia. É típica do senso comum a crença de que os autores devem evitar a primeira pessoa do singular ou plural, por exemplo, não aceitando as expressões "Eu analisei/nós analisamos". Em linhas gerais, acredita-se que formas que evidenciem a presença autoral e a emissão de juízos de valor devem ser evitadas.

Hyland (1998, 2002, 2005, 2011, 2018), na contramão dos pressupostos difundidos por esse senso comum, salienta que a escrita acadêmica é o espaço de constantes negociações com o outro e de tomada de decisões que criam efeitos nos leitores, atestando que escrever como um perito é uma prática que envolve conhecimento de todo um sistema semiótico de valores e recursos retóricos dentro da área de atuação. A elaboração dos textos é uma forma de construção do pesquisador, uma aquisição gradual dos recursos necessários para clamar por pertencimento em uma comunidade ao mesmo tempo em que se estabelece certo espaço individual.

Como continuação desse raciocínio, Hyland (2018) afırma que as possibilidades de elementos linguísticos e retóricos variam enormemente entre as áreas e disciplinas, isso porque elas expressam práticas sociais e epistemológicas muito distintas, relacionando a 
prática profissional com os discursos correntes - "estudantes aprendem suas disciplinas conforme aprendem seus discursos" (HYLAND, 2018, p. 391, tradução nossa²).

A tese de Hyland (2018) já se provou válida em pesquisas posteriores. Silva (2014) observou que os autores de artigos acadêmicos da área de Linguística tendem a utilizar mais a automenção (uso de eu/nós) em seus textos, enquanto os pesquisadores da área de Letras não se utilizam muito desse recurso. Em contrapartida, eles destacam a presença do leitor, criando diálogos diretos com eles, "um 'chamamento' para que a audiência participe das discussões apresentadas" (SILVA, 2014, p. 112).

Gil e Aranha (2017) destacam que a análise de dois periódicos distintos da área de Antropologia - um voltado à Antropologia Social e em contato com outras disciplinas de Humanidades; e outro sobre Paleontologia e em intersecção com disciplinas exatas e biológicas, revelou materializações discrepantes - o primeiro utilizou mais recursos linguísticos de negociação do que o segundo, mesmo que ambos sejam da mesma área de pesquisa.

Pontes, Carvalho e Almeida. (2020), por meio de análise de elementos linguísticos em teses acadêmicas, afirma que os resumos das áreas de Ciência da Computação e Medicina tendem a apagar mais o sujeito de seu texto do que a área de Linguística Aplicada. Além disso, foi possível ver estilos individuais de escrita na Linguística Aplicada, fenômeno não manifestado nas outras áreas.

Consonante com os pressupostos defendidos acima, Becker e Trowler (2001) advogam que a forma pela qual os diferentes grupos acadêmicos se engajam com suas atividades profissionais é diretamente relacionada com as atividades intelectuais que desempenham, e isso, consequentemente, reflete-se na forma pela qual a escrita se constitui. Os pesquisadores ainda defendem que é necessário extrapolar as divisões Ciências Humanas e Sociais vs. Ciências Exatas e Biológicas, agregando a elas outras facetas que podem aproximá-las ou distanciá-las.

Se agregarmos às disciplinas o viés de natureza pura - não aplicadas diretamente a problemas do mundo exterior - e aplicada - regulada por influências externas e questões práticas do mundo real -, por exemplo, é possível que uma disciplina humana-pura se aproxime mais em comportamento de disciplinas exatas-puras, do que humanasaplicadas. Abre-se um leque de possibilidades novas na maneira de se enxergar as disciplinas e suas fronteiras com outras.

2 No original: "students learn their disciplines as they learn its discourses.". 
Ainda que pesquisas sobre escrita acadêmica e a relação dela com as áreas de conhecimento sejam um solo fértil, a literatura aponta algumas brechas. Uma dessas brechas é a necessidade de mais investimento em pesquisas voltadas para a área de humanas e sua forma de escrita e discursos, conforme argumentado por Bhatia (2017), ao afirmar que há bastantes esforços de pesquisa em áreas como Direito, Medicina e Saúde, Ciências Contábeis, Ciência e Tecnologia, colocando as áreas de Humanidades em segundo plano.

Assim, com o propósito de suprir uma pequena brecha nos estudos de escrita acadêmica, notadamente nas Humanidades, o objetivo deste artigo é, por meio da análise da superfície textual e de as características dos escopos das revistas estudadas, analisar 48 abstracts, publicados em quatro revistas internacionais de Ciência Política e Linguística com a finalidade de observar (i) como o metadiscurso ${ }^{3}$ aparece na constituição da escrita acadêmica em disciplinas de Humanidades, mais especificamente Linguística e Ciência Política ${ }^{4}$, e (ii) se é possível notar algum padrão de semelhança e/ou diferença nas recorrências do metadiscurso entre as duas disciplinas, considerando suas características e suas fronteiras disciplinares.

\section{A Análise de Gênero Crítica}

Considerando o processo de escrita como forma de pertencimento a uma área específica, conforme discutido na introdução deste artigo, é possível construir uma ponte entre essa característica e a da Análise de Gênero Crítica, proposta por Bhatia (2017).

A abordagem de Bhatia (2017) propõe que o discurso acadêmico-profissional deve ser analisado em quatro níveis distintos e sobrepostos, compondo uma visão holística do processo de escrita e de interação.

O primeiro nível - discurso como texto - refere-se às propriedades da superfície textual, incluindo características funcionais do discurso, como aspectos léxico-gramaticais, semânticos e estruturais do texto. Esse nível, normalmente, exclui análises mais profundas de contexto, ignorando o papel do escritor ou do leitor na construção e interpretação do texto.

O segundo nível - discurso como gênero - avança a análise textual ao incorporar o contexto e as formas pelas quais o texto é construído, interpretado, usado e explorado em contextos sociais, institucionais ou profissionais particulares para atingir propósitos específicos. Nesse nível, são conduzidas análises linguísticas, sociopragmáticas e

3 A noção de metadiscurso será discutida nas seções subsequentes.

4 Os critérios de seleção para o corpus de análise serão apresentados na seção de metodologia. 
etnográficas (confira SWALES, 1990; BHATIA, 1993; SWALES, 2004), sendo uma das formas de análise mais populares nas aplicações pedagógicas de Inglês para Fins Específicos.

A prática profissional, nível 3, contempla fatores como as escolhas de determinados gêneros para atingir objetivos específicos, e as formas apropriadas e efetivas de comunicação relativas a cada gênero. Consideram-se também os procedimentos discursivos, fatores associados com participantes autorizados a fazer contribuições válidas e apropriadas, mecanismos de participação, tipo de contribuição que um participante é permitido a fazer, entre outros. As práticas profissionais e os procedimentos discursivos fazem parte do contexto das disciplinas e culturas profissionais a qual um gênero pertence, chegando ao quarto nível - culturas profissionais.

O quarto nível, por sua vez, determina os limites e as restrições do contexto, como normas e convenções genéricas, objetivos da disciplina e da profissão, e as identidades profissionais, organizacionais e disciplinárias.

A análise de metadiscurso, conforme desenvolvida na seção a seguir, pode ser considerada uma das formas de englobar todos os níveis de análise propostos por Bhatia (2017), uma vez que, partindo da superfície textual, possibilita entender algumas das crenças e das práticas que regem determinada profissão e/ou disciplina.

\section{O metadiscurso e seu papel na construção dos discursos disciplinares}

O metadiscurso pode ser definido como o "conjunto de traços característicos que, quando vistos juntos, ajudam a explicar as interações entre os produtores e seus textos e entre os produtores e usuários" (HYLAND, 2005, p. 125). Para Hyland (2015), há dois tipos de recursos - interativos e interacionais - que permitem a análise do metadiscurso e que podem ser de grande valia para se observar como a escrita acadêmica é construída, principalmente, considerando as disciplinas em que ocorrem.

Os recursos interativos se voltam para a forma pela qual o discurso é organizado, a fim de antecipar determinado tipo de conhecimento no leitor, permitindo ao autor gerenciar as informações para estabelecer qual interpretação ele deseja. Já os recursos interacionais se referem aos esforços do escritor em dosar sua personalidade no texto e construir uma relação apropriada com seus dados, argumentos e público. De acordo com sua função principal no texto, esses recursos são divididos em duas instâncias: 
(i) Stance: ${ }^{5}$ relacionado com a voz "autoral". É a forma pela qual o autor se mostra no texto, como apresenta suas opiniões, julgamentos e comprometimentos.

(ii) Engagement: o foco é a presença do leitor. São as marcas discursivas que mostram a forma pela qual o escritor reconhece o leitor, e o engaja no texto, guiando-o ao longo das argumentações e interpretações.

Cada uma dessas instâncias ainda se subdivide em outras. Dentre todos os recursos propostos por Hyland (2015) e passíveis de serem analisados, este artigo desenvolve uma análise de stance, mais especificamente hedges e boosters, recursos que permitem ao pesquisador o posicionamento de sua voz autoral.

Os hedges são dispositivos linguísticos que permitem ao autor modalizar determinada informação como uma opinião, uma interpretação e não como um fato. Os usos de palavras como might (poderia), perhaps (talvez) e possible (possível), por exemplo, são formas de abrandar o discurso. Já os boosters, ao contrário do primeiro, são usados para exprimir certeza sobre alguma proposição ou opinião. São vocábulos e expressões como must (deve), for sure (com certeza), without doubt (sem dúvidas).

Ao aplicar os conceitos acima em um corpus de 240 artigos de pesquisa, divididos entre oito disciplinas do conhecimento, Hyland $(2005,2011)$ observa que há grandes diferenças entre as disciplinas de Ciências Humanas e Ciências Sociais das de Ciências Exatas e Ciências Biológicas, e que essas diferenças refletem a forma como cada uma concebe pesquisa e discurso acadêmico, traçando, portanto, considerações que atingem todos os níveis de discurso proposto por Bhatia (2017).

Nas considerações do pesquisador (HYLAND, 2005, 2011), as Ciências Humanas e Sociais, por exemplo, são mais interpretativas e influenciadas por fatores contextuais. Como há uma diversidade nos resultados de pesquisa e diversos pontos de vista sobre o mesmo objeto, há uma necessidade de promover um diálogo maior, por meio do reconhecimento de outras vozes. Há um esforço em estabelecer credibilidade e criar um entendimento com os leitores.

Esse traço constitutivo é confirmado pela maior presença de hedges e boosters do que nas disciplinas exatas, por exemplo. Os hedges permitem que o autor seja mais cauteloso, sem impor somente uma explicação para determinado fato, enquanto os boosters ajudam a estabelecer o significado da pesquisa.

5 Optou-se em manter os termos originais, em língua inglesa, devido à tradição da abordagem escolhida para fundamentação do artigo. 
O autor ainda explica que, nas Engenharias e nas Ciências Exatas, a crença é de que o texto e os fatos devem falar por si só, levando os autores a privilegiar uma objetividade linguística, desconsiderando posicionamentos mais interpretativos. Hyland (2011) também aponta que, nessas áreas, os pesquisadores dividem um conhecimento compartilhado mais extenso, uma vez que é mais comum partirem de uma mesma revisão literária e se calcarem em métodos de pesquisa idênticos ou similares. Dessa forma, diminui-se o esforço retórico necessário para convencer os leitores da validade de seu território de investigação e, consequentemente, a necessidade do uso de modalizadores do discurso.

Logo, por não ser necessário um forte elemento interpessoal, as áreas duras permitem uma visão mais impessoal e indutiva da ciência, uma visão que mostra o cientista "descobrindo" a verdade e não a construindo. Apesar de haver o uso de hedges e boosters, eles estão presentes em menor quantidade do que nas Ciências Humanas e Sociais, como uma forma de diminuir o papel do escritor e garantir uma escrita mais impessoal.

Apresentados os embasamentos teóricos que norteiam essa pesquisa - a análise de gênero crítica (BHATIA, 2017), as fronteiras disciplinares (BECKER; TROWLER, 2001) e o metadiscurso, especificamente hedges e boosters (HYLAND, 1998, 2002, 2005, 2011, 2018) - a próxima seção expõe os procedimentos de coleta e de análise utilizados neste artigo.

\section{Metodologia}

A fase metodológica que compôs essa pesquisa dividiu-se em três etapas: o estabelecimento dos critérios para coleta dos periódicos e abstracts que comporiam o corpus de análise, investigação do perfil do corpus coletado e, por fim, a análise textual do corpus.

\section{Procedimentos de coleta}

Para a realização da pesquisa, foram escolhidas duas disciplinas da área de Humanidades para a análise do metadiscurso, a saber, Ciência Política e Linguística. Ambas foram escolhidas a partir da área de inserção da pesquisadora em suas práticas acadêmicas, no caso da segunda, ou por interesse pessoal, no caso da primeira.

De cada disciplina escolhida, foram selecionados dois periódicos distintos para a coleta de abstracts, gênero acadêmico considerado o cartão de visitas de um artigo científico, já que "daqueles que leem o resumo, apenas alguns leem o artigo" (SWALES, 1990, p. 179, tradução nossa) ${ }^{6}$, justificando sua escolha devido sua importância para a leitura do manuscrito completo.

6 No original: "[...] and of those who read the abstract only some will read the article itself.". 
Os critérios utilizados para a seleção das revistas e abstracts foram:

- Todos os periódicos deveriam ser publicados em língua inglesa (língua franca da ciência e idioma da maioria dos periódicos com maior prestígio científico);

- Todos os periódicos deveriam ser Qualis A1, de acordo com a classificação realizada pela Capes e apresentar alto fator de impacto;

- Todos os periódicos deveriam apresentar banco de dados on-line;

- Todos os periódicos deveriam apresentar em seu banco de dados um ranking de artigos mais citados dentro da publicação, uma vez que a escolha dos abstracts de artigos a serem analisados foi determinada pela sua importância/impacto.

Foram selecionados, com base nos critérios:

Quadro 1. Organização do corpus

\begin{tabular}{|c|c|c|c|}
\hline \multirow{2}{*}{ Disciplina } & Periódico & Abreviação & Abstracts \\
\hline \multirow{2}{*}{ Ciências Políticas } & The American Political Science Review & APSR & P1 a P12 \\
\cline { 2 - 4 } & Journal of Politics & JP & P13 a P24 \\
\hline \multirow{2}{*}{ Linguística } & Brain and Language & BL & L1 a L12 \\
\cline { 2 - 4 } & Journal of Phonetics & JPh & L13 a L24 \\
\hline
\end{tabular}

Fonte: Elaboração própria (2020)

O quadro acima mostra os quatro periódicos escolhidos para a análise divididos pelas disciplinas, as abreviações que serão usadas na análise, assim como a organização dos 48 abstracts que compõem o corpus - 24 abstracts por disciplina, 12 abstracts por periódico.

\section{Natureza do corpus}

A Tabela 1 apresenta as principais informações a respeito da natureza do corpus que são de fundamental importância para a análise dos resultados. 
Tabela 1. Natureza do corpus

\begin{tabular}{|c|c|c|c|}
\hline Periódico & Editora & Qtd de palavras & Outras áreas de avaliação \\
\hline APSR & Cambridge & 1885 & - \\
\hline JP & Cambridge & 1636 & - \\
\hline BL & Elsevier & 2575 & Neuropsicologia (A1), Neurociência (A1) \\
\hline JPh & Elsevier & 2345 & Fala e audição (A1) \\
\hline
\end{tabular}

Fonte: Elaboração própria (2020)

Há duas características do corpus apresentadas no quadro. A primeira é a quantificação do corpus em relação à quantidade de palavras. A contagem das palavras foi realizada pela ferramenta do programa Word que informa a quantidade de palavras e de caracteres presentes em trechos selecionados de um arquivo ou em sua totalidade. As 8.441 palavras totais do corpus estão divididas pelos periódicos conforme apresentado. Outra característica são as áreas de avaliação nas quais as revistas receberam Qualis, além de suas respectivas disciplinas, e as políticas editoriais que guiam os autores na elaboração dos artigos e de seus abstracts.

\section{American Political Science Review (APSR)}

A publicação American Political Science Review é feita pelo Cambridge Journals Online e sua circulação data do ano de 1906. Seu fator de impacto é 3.05. Os principais temas de interesse da revista são pesquisas relacionadas com Teoria Política, Política Americana, Políticas Públicas, Administração Pública, Política Comparada e Relações Internacionais, ou seja, apresenta escopo de pesquisa amplo e abrangente dentro da Ciência Política. Apresenta avaliação em Qualis apenas para Ciência Política e Relações Internacionais, de acordo com o Capes periódicos.

\section{The Journal of Politics (JOP)}

A publicação The Journal of Politics também é uma publicação da Cambridge Journals Online e seu formato em papel pela Cambridge University Press desde o ano de 1938. Seu fator de impacto é 1,478.

O periódico apresenta foco de interesse bem abrangente para as pesquisas, citando algumas das áreas de interesse, tais como, pesquisas em Política Comparada, Relações Internacionais, Administração Política, entre outras, aproximando-se de APSR uma vez que ambos se interessam pelas diversas abordagens, subdivisões e linhas de pesquisa disponíveis dentro da disciplina. Da mesma forma que o periódico anterior, também apresenta avaliação em Qualis apenas em Ciência Política e Relações Internacionais. 


\section{Brain and Language (BL)}

A publicação Brain and Language é veiculada pela editora Elsevier e é uma publicação mensal. Seu fator de impacto é 3,115. O principal escopo de pesquisa da revista são os mecanismos neurobiológicos que subjazem a linguagem humana. Além disso, ainda coloca como interesse científico dados e perspectivas teóricas retirados da Linguística e Psicologia.

Ademais de um periódico Qualis A1 para a Linguística, ele também é considerado de alto fator de impacto para a Psicologia Cognitiva e Experimental, Neurologia, Neuropsicologia e Fisiologia. O Science Journal Report ainda classifica o periódico como de alto fator de impacto para as disciplinas de Neuropsicologia e Neurociência, reforçando a sua interface com os estudos biológicos.

\section{Journal of Phonetics (JPh)}

Journal of Phonetics também é uma publicação da editora Elsevier. Apresenta fator de impacto 1,410. O principal interesse da revista são os estudos de aspectos fonéticos da língua e os processos linguísticos da comunicação. O periódico lista uma série de áreas de interesse, tais como, aquisição de fala, aspectos fonéticos de aquisição de segunda língua, patologias fonéticas, entre outras. Também aceita trabalhos de outras áreas desde que com foco em aspectos fonéticos. Em relação ao Qualis, é classificado como A1 em Linguística e Estudos de fala/audição, colocando-se também em posição fronteiriça com as Ciências Biológicas e estudos da área da saúde.

\section{Metodologia de análise}

Definido o corpus de estudo, conforme apresentado nas seções anteriores, a metodologia de análise empregada na pesquisa foi baseada na lista de palavras de hedges e boosters elaborada e utilizada nas pesquisas de Hyland (2005).

Quadro 1. Lista de hedges e boosters analisados no corpus

\begin{tabular}{|l|l|l|l|}
\hline \multicolumn{5}{|c|}{ Hedges } \\
\hline About & Claims & In most instances & Perhaps \\
\hline Claims & Could & In my view & Plausible \\
\hline Almost & Could not & In our opinion & Plausibly \\
\hline Apparent & Doubt & In our view & Possible \\
\hline Apparently & Doubtful & In this view & Possibly \\
\hline
\end{tabular}




\begin{tabular}{|c|c|c|c|}
\hline Appear & Doubtless & Indicate & Postulate \\
\hline Appeared & Essentially & Indicated & Postulated \\
\hline Appears & Estimate & Indicates & Postulates \\
\hline Approximately & Estimated & Interpret & Presumable \\
\hline Argue & Estimates & Interprets & Presumably \\
\hline Argued & Fairly & Largely & Probable \\
\hline Argues & Feel & Least & Probably \\
\hline Around & Feels & Likely & Propose \\
\hline Assume & Felt & Mainly & Quite \\
\hline Assumed & Frequently & May & Rare \\
\hline Assumes & From my perspective & Maybe & Rarely \\
\hline Assumption & From our perspective & Might & Rather \\
\hline Broadly & From this perspective & More or less & Relatively \\
\hline Certain amount & General & Most & Roughly \\
\hline Certain extent & Generally & Mostly & Seems \\
\hline Certain level & Guess & Not necessarily & Seem \\
\hline Certain that & In general & Often & Sense \\
\hline Certainly & In most cases & On the whole & Should \\
\hline Claim & In my opinion & Ought & Sometimes \\
\hline Claimed & & & \\
\hline \multicolumn{4}{|c|}{ Boosters } \\
\hline Actually & Decidedly & In fact & Obviously \\
\hline Always & Definitely & Incontestable & Of course \\
\hline Believe & Definite & Incontestably & Predict \\
\hline Believed & Demonstrate & Incontrovertible & Prove \\
\hline Believes & Demonstrated & Incontrovertibly & Proved \\
\hline Beyond doubt & Demonstrates & Indeed & Proves \\
\hline Certain & Determine & Indisputable & Realize \\
\hline
\end{tabular}




\begin{tabular}{|l|l|l|l|}
\hline Certainly & Doubtless & Indisputably & Realized \\
\hline Clear & Establish & Inevitable & Realizes \\
\hline Clearly & Established & Know & Really \\
\hline Conclude & Evidence & Known & Show \\
\hline Conclusively & Evident & Must & Showed \\
\hline Confirm & Evidently & Necessarily & Shows \\
\hline Consistent with & Find & Never & Shown \\
\hline Convincing & Finds & No doubt & Sure \\
\hline Convincingly & Found & Obvious & Surely \\
\hline
\end{tabular}

Fonte: Hyland (2005, p. 223-224)

Como a análise de hedges e boosters parte do uso ou não de vocábulos específicos, ou seja, da materialização da superfície textual, consideramo-la como representativa do primeiro nível de discurso proposto por Bhatia (2017) - discurso como texto.

As palavras acima, indicadas por Hyland (2005), foram buscadas por meio do programa WordSmith Tools, versão 5.0, mais especificamente pela ferramenta Concord, que permite a busca específica de itens lexicais dentro de um corpus. Após a busca pela recorrência dos termos, os resultados foram quantificados em gráficos divididos por periódicos e analisados à luz dos preceitos teóricos discutidos.

\section{Análise e discussão de dados}

A análise linguística proposta neste trabalho é calcada nos estudos de Hyland (1998, $2002,2005,2011$ ) e seu postulado de que o metadiscurso, mais especificamente hedges e boosters neste artigo, oferece pistas sobre a forma pela qual as disciplinas concebem o fazer científico.

De os 115 hedges e 80 boosters listados por Hyland (2011), foram encontrados, respectivamente 55 e 37 termos. Dentre todos os vocábulos analisados, destacam-se pela maior presença no corpus, independentemente da disciplina, os hedges suggest (sugerir) e suas conjugações de pessoas e tempos verbais (24 exemplos), most (mais, em 23 exemplos), may (pode, em 22 exemplos) relatively (relativamente, em 10 exemplos), argue, could, rather e would (discutir, poderia, preferencialmente, em 8 exemplos cada). Quanto aos boosters, destacam-se show/shows/showed (mostrar, mostra, mostrou, com 44 exemplos), evidence (evidência, em 21 exemplos), demonstrate e suas variações de 
pessoa e tempo (demonstrar, em 12 exemplos) e consistente with (consistente com, 9 exemplos).

Em linhas gerais, tais termos foram empregados mais nas sentenças que apresentam os resultados da pesquisa no abstract. O resultado mostra o novo da pesquisa, logo um maior uso de stance se justifica pela necessidade de o autor convencer o leitor de suas interpretações, tanto por meio de estratégias que fortificam o apresentado, aproximando-o de um fato, quanto por meio da modalização do que está sendo dito, em um movimento de prevenção de possíveis discordâncias, como observado no exemplo abaixo e representativo da dinâmica do uso desses recursos no corpus:

L11: Three of these four patients showed no left frontal activity by completion of treatment, indicating that right posterior lateral frontal activity supported category-member generation. Patients who improved in treatment showed no difference in lateralization of lateral frontal activity from normal controls pre-treatment, but post-treatment, their lateral frontal activity during categorymember generation was significantly more right lateralized than that of controls. Patterns of activity pre- and post-treatment suggested increasing efficiency of cortical processing as a result of treatment in the four patients who improved. The one patient who did not improve during treatment showed a leftward shift in lateral frontal lateralization that was significantly different from the four patients who did improve. Neither medial frontal nor posterior perisylvian re-lateralization from immediately pre- to immediately post-treatment images was a necessary condition for significant treatment gains or shift in lateral frontal lateralization. Of the three patients who improved and in whom posterior perisylvian activity could be measured at post-treatment fMRI, all maintained equal or greater amounts of left-hemisphere perisylvian activity as compared to right. This finding is consistent with reviews suggesting both hemispheres are involved in recovery of language in aphasia patients.

O exemplo, retirado do periódico Brain and Language, alterna o uso de hedges e boosters, a fim de alcançar intenções particulares. Os boosters, neste caso showed e consistent with, foram utilizados na apresentação dos achados de pesquisa: "quatro pacientes não mostraram atividade frontal do lado esquerdo", "pacientes que melhoraram não mostraram diferença na lateralização". Ou seja, os fortificadores foram empregados de forma a apresentar fatos que não poderiam ter outra leitura possível por se tratarem de dados recorrentes de procedimentos laboratoriais e uso de equipamentos.

Os hedges, de outra forma, foram usados no momento de apresentar possíveis interpretações dos autores para os fatos mencionados: "os padrões de atividades pré e pós-tratamento sugeriram eficiência melhorada nos processos corticais como resultado do tratamento nos quatro pacientes que melhoraram". Percebe-se um movimento de 
preservação, já que, por se tratar de uma interpretação, e não de um fato fruto de um procedimento laboratorial, por exemplo, pode gerar, mais facilmente, discordância por parte do leitor.

De maneira semelhante ao exemplo L11, P5, provindo da publicação American Political Science Review, também apresenta uso de stance nos resultados de forma a criar um equilíbrio entre os movimentos de preservação e de reafırmação. O hedge indicate (indicar) possibilita a apresentação dos resultados de forma a deixar uma possibilidade de discordância para o leitor a respeito da importância da genética na influência de atitudes políticas do indivíduo, sem o rechaço à leitura dos autores, enquanto o booster conclude (concluir) permite a apresentação da conclusão da pesquisa de forma mais firme.

P5: The results indicate that genetics plays an important role in shaping political attitudes and ideologies but a more modest role in forming party identification; as such, they call for finer distinctions in theorizing about the sources of political attitudes. We conclude by urging political scientists to incorporate genetic influences, specifically interactions between genetic heritability and social environment, into models of political attitude formation.

Em todos os abstracts analisados, hedges e boosters foram encontrados. A razão que gera discrepância entre as disciplinas não é sua presença ou ausência, mas sim a quantidade de vezes em que apareceram no corpus. Das 1.885 palavras totais dos abstracts de APSR, $3,12 \%$ enquadram-se na categoria de hedges e boosters; em CA, das 1.636 palavras totais, $3,02 \%$ estão nessa categoria. Ambos os periódicos de Linguística apresentam um número maior de palavras - 2.575, em BL, e 2.345, em JPh - e um número de stance menor comparado com os periódicos de Ciência Política - 1,55\% e 2,55\%, respectivamente. Esses dados podem ser visualizados com mais facilidade no gráfıco abaixo:

Gráfico 1. Quantificação de stance por periódico

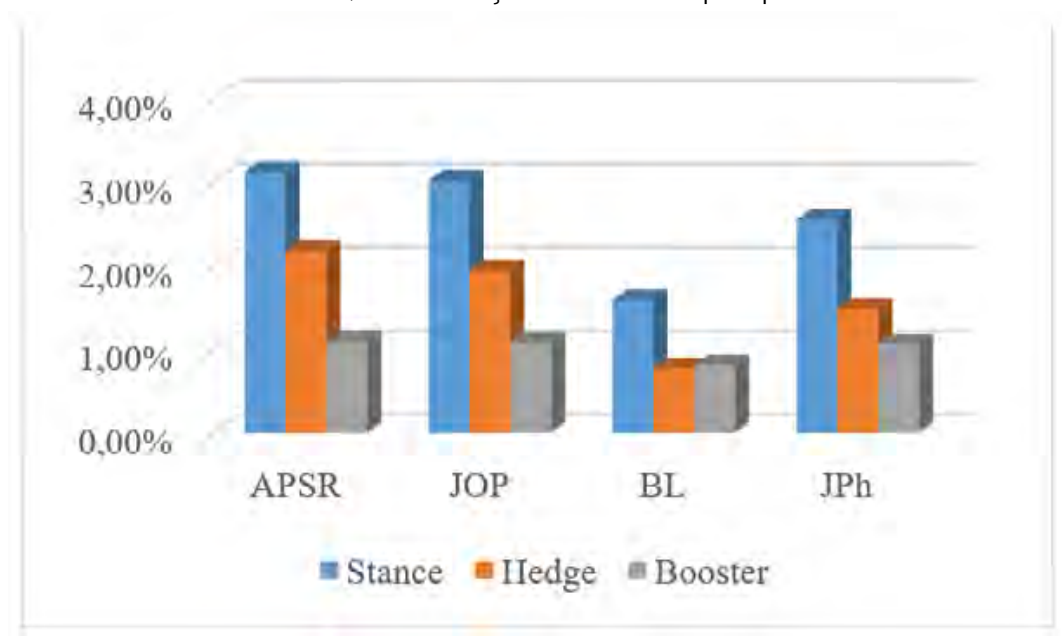

Fonte: Elaboração própria (2020) 
Se quantificarmos as recorrências pelas disciplinas, tem-se:

Gráfico 2. Quantificação de stance por disciplina

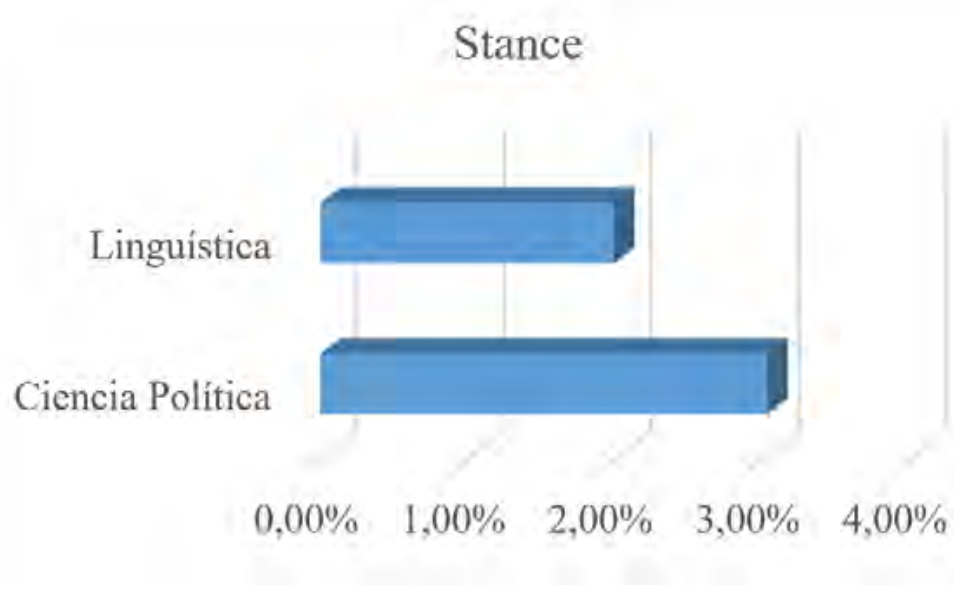

Fonte: Elaboração própria (2020)

Ao considerarmos as pesquisas anteriores de Hyland (1998, 2002, 2005, 2011), pode-se afirmar que os dados encontrados no corpus de análise não se enquadram totalmente nas considerações feitas pelo pesquisador. Pode-se pontuar como ponto de intersecção entre esta pesquisa e as de Hyland $(1998,2002,2005,2011)$, a maior recorrência de hedges do que de boosters, de maneira geral. Ainda assim, BL foge dessa estatística a ser o único periódico com maior recorrência de boosters, contrariando os achados anteriores de pesquisa de que os hedges são mais recorrentes. Ademais, o uso maior de fortificadores é característico das Ciências Exatas e Biológicas, não das Ciências Humanas e Sociais.

Ao compararmos as porcentagens de stance entre as disciplinas e os periódicos, vê-se que, ainda que ambas as disciplinas sejam de Humanidades, Ciência Política apresenta quase o dobro a mais de recursos interacionais do que Linguística. Só é possível encontrar possíveis explicações para essa materialização linguística no corpus se unir a ela a questão das fronteiras disciplinares.

O argumento principal feito aqui para justificar os achados do corpus é que, apesar de dividirem esse mesmo território e partirem de um ponto comum, cada periódico estudado ocupa diferentes fronteiras com outras disciplinas e áreas, o que agrega características pertencentes a outros domínios. Os escopos de pesquisa apresentados nos periódicos e as avaliações de Qualis mostram que as revistas se colocam em fronteiras diferentes, como na imagem abaixo: 
Figura 1. Fronteiras disciplinares no corpus

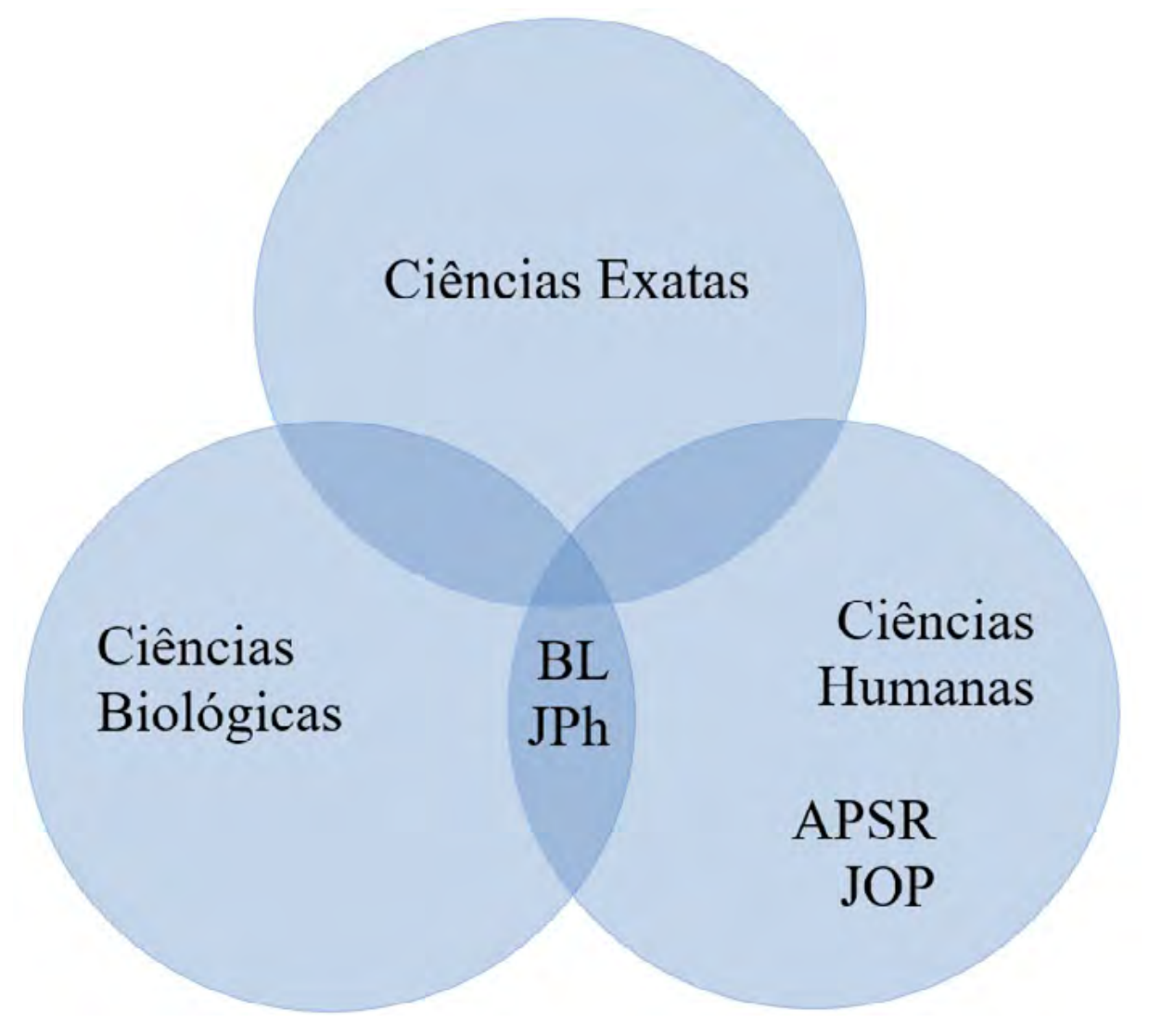

Fonte: Elaboração própria (2020)

Ambos os periódicos de Ciência Política, como já discutido na seção de metodologia, apresentam interesse de pesquisa abrangente dentro da área e não se aproximam de fronteiras com outras áreas do conhecimento. Em contrapartida, as revistas de Linguística têm escopo de pesquisa bem específico em interface clara com disciplinas das áreas da Saúde e da Biologia, ocupando uma fronteira distinta.

Os periódicos de Linguística, portanto, aproximam-se da realização metadiscursiva das Ciências Exatas e Biológicas ao apresentar menor uso de recursos interativos se comparado com a outra disciplina analisada, e se vale da fortificação dos boosters no discurso com mais frequência. Essa materialização mostra uma busca maior por um discurso que não centralize a figura do pesquisador como o agente principal da pesquisa, revelando a crença na busca pela objetividade da ciência. Logo, uma escrita acadêmica de sucesso para esses periódicos deve privilegiar o traço da objetividade, sendo esta constitutiva da identidade profissional buscada pelas revistas. 
Dessa forma, por meio da análise da superfície textual, nível um proposto por Bhatia (2017), aliada à observação do escopo de pesquisa dos periódicos analisados, conforme apresentado na metodologia acerca das características de cada periódico e sua intersecção com outros campos do saber, foi possível tecer considerações acerca de algumas das crenças que permeiam os fazeres científicos analisados, ou seja, vislumbrar as culturas profissionais, quarto nível de análise proposto por Bhatia (2017), relacionadas aos artigos e às revistas analisadas, confirmando a tese do autor de que os níveis do discurso são sobrepostos e indissociáveis.

\section{Considerações finais}

Todas as disciplinas são heterogêneas em sua natureza. Elas não são orientadas por apenas uma teoria, uma metodologia e uma forma de fazer pesquisa. Ao contrário, as Humanidades, enfoque deste artigo, são palco de debates e embates entre diversas perspectivas e conseguem estudar o mesmo objeto, o ser humano, de formas bastante distintas.

Essas características se repetem dentro de cada disciplina. As duas disciplinas estudadas apresentam subdivisões em orientações e em linhas de pesquisas, cada qual responsável por fragmentar ainda mais o objeto de estudo das Humanas e estudá-lo de forma cada vez mais específica. Essas subdivisões fazem com que elas se posicionem em locais diferentes, fazendo fronteiras com outras áreas e disciplinas.

As fronteiras das Ciências Humanas são mais permeáveis e osmóticas, permitindo uma troca maior com o que está ao seu redor e isso se reflete na constituição e na materialização da escrita. Observou-se que, ainda que as duas disciplinas analisadas sejam pertencentes ao domínio das Humanidades, os periódicos trazem recortes e escopos de pesquisas diferentes, revelando fronteiras disciplinares distintas e resultando em um uso diferente do metadiscurso.

O fato de os jornais de Ciência Política não apresentarem diferenciação no escopo de pesquisa, colocando-se em uma mesma fronteira, cujo contato primordial é da disciplina com outras de Humanidades, diferenciou-o das publicações de Linguística que, por apresentarem forte intersecção com disciplinas biológicas, por exemplo, Neurologia e Fisiologia, revelaram uma materialidade linguística mais próxima da esperada em Ciências Exatas e Biológicas. As fronteiras, portanto, como discutido por Becher e Trowler (2001), provam ser uma questão de grande importância nos estudos de escrita acadêmica.

Coincidentemente, as duas publicações das disciplinas apresentaram mesma vizinhança disciplinar. Seria uma possibilidade, dentro da mesma disciplina, encontrar periódicos com materializações distintas, como descrito por Gil e Aranha (2017) 
A partir da superfície textual encontrada no gênero abstract - nível 1 da análise proposta por Bhatia (2017) - aliada à observação do contexto das características dos periódicos, foi possível acessar algumas das crenças do fazer pesquisa de cada disciplina, notadamente do recorte proposto pelos periódicos. Dessa forma, prova-se verdadeira para esta pesquisa a disposição de Bhatia (2017) da indissociação dos níveis do discurso.

\section{Agradecimentos}

Agradeço à Fundação de Amparo à Pesquisa do Estado de São Paulo (FAPESP) pelo auxílio à pesquisa e às professoras doutoras Lilia Abreu-Tardeli e Solange Aranha pelas contribuições ao trabalho.

\section{REFERÊNCIAS}

AMERICAN POLITICAL SCIENCE REVIEW. Disponível em: https://www.cambridge.org/ core/journals/american-political-science-review. Acesso em: 08 dez. 2018.

BECHER, T.; TROWLER, P. Academic tribes and territories: intellectual enquiry and the culture of disciplines. 2. ed. Buckingham: Society for Research into Higher Education \& Open University Press, 2001.

BHATIA, V. K. Analysing genre: language use in professional settings. UK: Longman Group, 1993.

BHATIA, V. K. Critical Genre Analysis: Investigating Interdiscursive Performance in Professional Practice. London: Routledge, 2017.

BRAIN AND LANGUAGE. Disponível em: https://www.journals.elsevier.com/brain-andlanguage. Acesso em: 08 dez. 2018.

GIL, B. O gênero acadêmico: resumos nas áreas de Antropologia, Linguística e Ciência Política. 2014. Dissertação (Mestrado em Estudos Linguísticos) - Instituto de Biociências, Letras e Ciências Exatas, Universidade Estadual Paulista "Júlio de Mesquita Filho", São José do Rio Preto, 2014.

GIL, B.; ARANHA, S. Um estudo do gênero abstract na disciplina de Antropologia: a heterogeneidade da(s) área(s). D.E.L.T.A., v. 33.3, p. 843-871, 2017.

HYLAND, K. Boosting, hedging and the negotiation of academic knowledge. TEXT 18, v. 3, p. 349-382, 1998. 
HYLAND, K. Directives: Argument and Engagement in Academic Writing. Applied Linguistics, v. 23/2, p. 215-239, 2002.

HYLAND, K. Metadiscourse - exploring interaction in writing. London: Continuum, 2005.

HYLAND, K. Disciplines and Discourse: Social Interactions in Construction of Knowledge. In: STARKE-MYERRING, D.; PARÉ, A.; ARTEMEVE, N.; HORNE, M.; YOUSOUBOVA, L. (ed.). Writing in knowledge societies. Perspectives on Writings. Fort Collins, Colorado: The WAC Clearinghouse and Parlor Press, 2011. p. 193-214.

HYLAND, K. Genre and Second Language Writing. The TESOL Encyclopedia of English Language Teaching. Hoboken, NJ: Wiley-Blackwell, 2018. p. 2359-2364.

JOURNAL OF PHONETICS: Disponível em: https://www.journals.elsevier.com/journal-ofphonetics. Acesso em: 08 dez. 2018.

PONTES, A. L.; CARVALHO, D. N.; ALMEIDA, E. C. Recursos metadiscursivos em resumos de tese: o estilo em textos especializados. Forum lingüístico, Florianópolis, v. 17, n. 4, p. 5381-5397, out./dez. 2020.

SILVA, A. C. O metadiscurso em artigos científicos de Linguística e Literatura. 2014. Dissertação (Mestrado em Linguística) - Universidade Federal de Pernambuco, Centro de Artes e Comunicação, Recife, 2014.

SWALES, J. Genre analysis: English in academic and research settings. Cambridge: Cambridge University Press, 1990.

SWALES, J. Research genre: Explorations and Aplications. Ann Arbor: The University of Michigan Press, 2004.

THE JOURNAL OF POLITICS. Disponível em: https://www.cambridge.org/core/journals/ american-political-science-review. Acesso em: 08 dez. 2018. 